\title{
VIRTUALIZACIÓN DE INSTRUMENTOS DE EVALUACIÓN DESTINADOS A ACTIVIDADES FUERA DEL AULA: CUESTIONARIOS EN RUTA DURANTE VISITAS A EMPRESAS DEL SECTOR QUÍMICO
}

\section{VIRTUALIZATION OF ASSESSMENT INSTRUMENTS FOR ACTIVITIES OUTSIDE THE CLASSROOM: QUESTIONNAIRES EN ROUTE DURING VISITS TO COMPANIES IN THE CHEMICAL SECTOR}

\author{
Carlos Pérez-Vicente, José Carlos Arrebola, Manuel Cruz, Ivana Pavlovic, Luis \\ Sánchez y Álvaro Caballero ${ }^{1}$
}

Fecha de recepción: 18/03/2019; Fecha de revisión: 27/05/2019; Fecha de aceptación: 08/07/2019

Cómo citar este artículo:

Pérez-Vicente, C., Arrebola, J.C., Cruz, M, Pavlovic, I., Sánchez, L. \& Caballero, Á. (2019). Virtualización de instrumentos de evaluación destinados a actividades fuera dl aula: cuestionarios en ruta durante visitas a empresas del sector químico. Revista de Innovación y Buenas Prácticas Docentes, 8(3), 88-100.

Autor de Correspondencia: alvaro.caballero@uco.es

\section{Resumen:}

En el proyecto desarrollado se ha buscado la implementación de herramientas m-learning que permitan la virtualización del proceso de evaluación de las competencias adquiridas por los estudiantes de Grado en las actividades realizadas fuera del aula, específicamente, visitas a empresas del sector químico. Gracias a este proyecto se ha pretendido sustituir la herramienta habitual de evaluación para este tipo de actividad (redacción de una memoria) por un instrumento interactivo tipo aplicación móvil -smartphones o tablets-. Esta virtualización permite al estudiante completar una evaluación tipo test una vez finalizada la actividad docente, aprovechando el tiempo del desplazamiento desde la planta industrial visitada hasta el punto de origen. Dos aplicaciones informáticas han sido evaluadas como soporte de las potenciales herramientas de evaluación (Kahoot y iSpringSuite), logrando alcanzar los objetivos propuestos en el proyecto.

Palabras clave: Ciencias, cuestionario virtual, herramienta evaluación, visitas empresas.

\begin{abstract}
:
In the developed project, the implementation of m-learning tools that allow the virtualization of the evaluation process of the competences acquired by the students of Degree in the activities carried out outside the classroom, specifically, visits to companies in the chemical sector. Thanks to this project, it has been tried to replace the usual evaluation tool for this type of activity (writing a report) with an interactive instrument such as a mobile application -smartphones or tablets-. This virtualization allows the student to complete a test type evaluation once the teaching activity has finished, taking advantage of the time of the trip from the industrial plant visited to the point of origin. Two computer applications have been evaluated as support for the potential assessment tools (Kahoot and iSpringSuite), achieving the objectives proposed in the project. Key Words: company visits, evaluation tool, Science, virtual questionnaire.
\end{abstract}




\section{INTRODUCCIÓN}

Las TICs están consideradas como las tecnologías más idóneas para promover la descentralización del aprendizaje del alumnado en los diferentes niveles educativos, y especialmente en el ámbito universitario, dado el mayor grado de experiencia tecnológica alcanzada en esta etapa (Canós \& Mauri, 2005; Canós, Canós \& Liern, 2008). La utilización de estas Tecnologías permite el acceso, creación, recreación, publicación, interconexión y construcción del conocimiento de forma distribuida y descentralizada. Además también posibilitan la formación de redes y permiten suplir adecuadamente la reducción de horas presenciales en los actuales planes.

La amplia disponibilidad de conectividad online (Wifi o conexión 3G-4G) ha permitido que la enseñanza universitaria llegue al alumno independientemente del lugar donde se halle, sin necesidad de requerir la presencia física en los diferentes Campus. Esta tipología de enseñanza, englobada comúnmente en el concepto conocido como "e-Learning", ofrece ventajas tales como que el estudiante puede acceder al curso en cualquier momento; elimina la necesidad de la presencia física del estudiante o profesor y reduce de manera considerable los costes requeridos derivados de la presencialidad, gracias a los métodos de enseñanza virtual a distancia.

Adicionalmente, se ha producido un avance espectacular en los últimos años en el uso de tabletas y teléfonos móviles de última generación (conocidos normalmente como "smartphones"), en ambos casos con conectividad online exclusiva de red Wifi o dual (adicional 3G-4G). Esta conectividad móvil ha permitido el desarrollo y utilización de un nuevo tipo de aprendizaje, diseñado específicamente para dispositivos móviles. De esta manera, a la disponibilidad de medios de aprendizaje "on-line" a través de un ordenador fijo o portátil (e-Learning), se ha sumado la aparición de medios de aprendizaje adaptados a las nuevas tecnologías móviles (m-Learning). La mayoría de los dispositivos móviles cuenta con un acceso online de datos continuo, bien a través de conexión wifi o redes de datos (3G-4G). Esto beneficia y facilita la implantación de aplicaciones adaptadas a esos dispositivos móviles para su ejecución "on-line".

En este marco, una herramienta fundamental de las TIC son las plataformas y aplicaciones digitales, posibilitando una fácil transferencia de conocimiento en el desarrollo de las actividades de los estudiantes (Ghemawat, 2017). Aun contando con esta importante ventaja, las plataformas digitales no suelen ser completamente aprovechadas en el contexto universitario dada su consideración de herramientas lúdicas, perdiendo la posibilidad de aprovechar las ventajas que ofrece para mejorar el proceso de enseñanza-aprendizaje (Pons, Bravo \& Ramírez, 2016). Kahoot es una de estas plataformas considerada como m-Learning, donde se pueden generar escenarios didácticos online para la resolución de ejercicios a través del uso de cuestionarios, videos, etc... El uso de esta herramienta virtual facilita la aplicación de los conocimientos en los procesos de enseñanza-aprendizaje (Guardia, et al., 2019; Gündüz \& Akkoyunlu, 2019; Martins, et al., 2019). 
Por otra parte, una herramienta importante para el currículum académico es el aprendizaje en campo. Para los estudiantes, las visitas a empresas relacionadas con el ámbito de su actividad académica suponen una alternativa complementaria a los sistemas tradicionales de enseñanza universitaria (Guenaga, \& Hernando, 2012). Las salidas a empresas se pueden encontrar como una de las preferencias del alumnado, apreciando el acercamiento a la realidad empresarial y entrando en contacto con los procesos productivos y las innovaciones. Las actividades fuera del aula como estas visitas a industrias o centros de producción pueden ser consideradas uno de los aspectos más importantes dentro del período de formación para cualquier estudiante, complementando su desarrollo profesional y personal (Mohamed, Pérez \& Montero, 2017).

Además, incrementan su capacidad de observación de los procesos productivos y avances tecnológicos en la organización. Logrando reafirmar los conocimientos teóricos con un panorama concreto sobre las necesidades actuales de la industria.

El proyecto ejecutado se enmarca dentro de este proceso de implantación de aplicaciones y desarrolla herramientas $m$-Learning que permiten la virtualización del proceso de evaluación de las competencias adquiridas por los estudiantes en las actividades realizadas fuera del aula, específicamente, visitas a empresas del sector Químico. Gracias a este proyecto se pretende en próximos cursos sustituir la herramienta habitual de evaluación para este tipo de actividad (redacción de una memoria) por un instrumento interactivo tipo aplicación móvil -destinado a ser empleado en smartphones o tablets-. Esta virtualización permitirá al estudiante completar una evaluación tipo test una vez finalizada la actividad docente, aprovechando el tiempo del desplazamiento desde la planta industrial visitada hasta el punto de origen. Con las consideraciones remarcadas, este proyecto se ha enmarcado por tanto en la línea de acción prioritaria "Procesos e instrumentos de evaluación".

El objetivo principal del Proyecto se centra en facilitar al alumnado y al profesorado una de las etapas de evaluación continua habitualmente usada en asignaturas que incluyen visita a industria entre sus actividades docentes. La virtualización de las herramientas de evaluación correspondientes a dichas visitas incentivará el aprendizaje provechoso y motivado del alumno y mejorará la captación de las competencias asignadas a esta metodología pedagógica. En este objetivo principal se enmarcan los hitos concretos:

-Desarrollo de herramientas para dispositivos móviles (m-Learning), fomentando la formación del profesorado en los procesos de virtualización de asignaturas.

-Interactividad virtual entre profesorado y alumnado a través de instrumentos online de evaluación de competencias.

Adicionalmente, remarcar que con el correcto desarrollo del Proyecto propuesto se cumplen varios de los objetivos marcados en el Plan de Innovación y Buenas Prácticas Docentes 2017-2018 de la UCO, como son las siguientes:

- Valorar y apoyar las prácticas docentes innovadoras que se consideran referentes de calidad.

- Potenciar la puesta en práctica de metodologías docentes centradas en la actividad del alumnado. 
- Apoyar la incorporación de las tecnologías de la información y comunicación, aplicadas a la enseñanza.

\section{DESARROLLO DE LA EXPERIENCIA DE INNOVACIÓN}

La metodología del Proyecto propuesto se ha basado en la selección, ensayo y utilización de las aplicaciones virtuales (app) para ser empleadas como herramientas de evaluación en actividades fuera del aula. Los elementos del trabajo interactivo están basados en el empleo de aplicaciones móviles de forma activa entre los profesores y los estudiantes, permitiendo plantear a lo largo de la actividad preguntas en las que todos los estudiantes tengan acceso en tiempo real y posibilidad de responder desde sus dispositivos móviles.

\subsection{Condiciones de aplicaciones móviles}

En el proceso de selección de aplicaciones a emplear para el desarrollo del Proyecto, la primera premisa ha consistido en garantizar la completa accesibilidad de la totalidad de los alumnos a la herramienta de evaluación. Para ello se ha analizado el uso de dispositivos móviles en la actualidad. Según la página web kantarworldpanel, más del 98\% del uso en España corresponde a dispositivos basados en sistemas operativos iOS y Android (Figura 1). Solamente una fracción minoritaria utiliza dispositivos con entornos operativos diferentes, tales como Java, Windows Phone o Symbian. De acuerdo con estos datos, se ha procedido a seleccionar herramientas virtuales que aseguren su compatibilidad con las versiones más actuales de los dos sistemas operativos indicados.
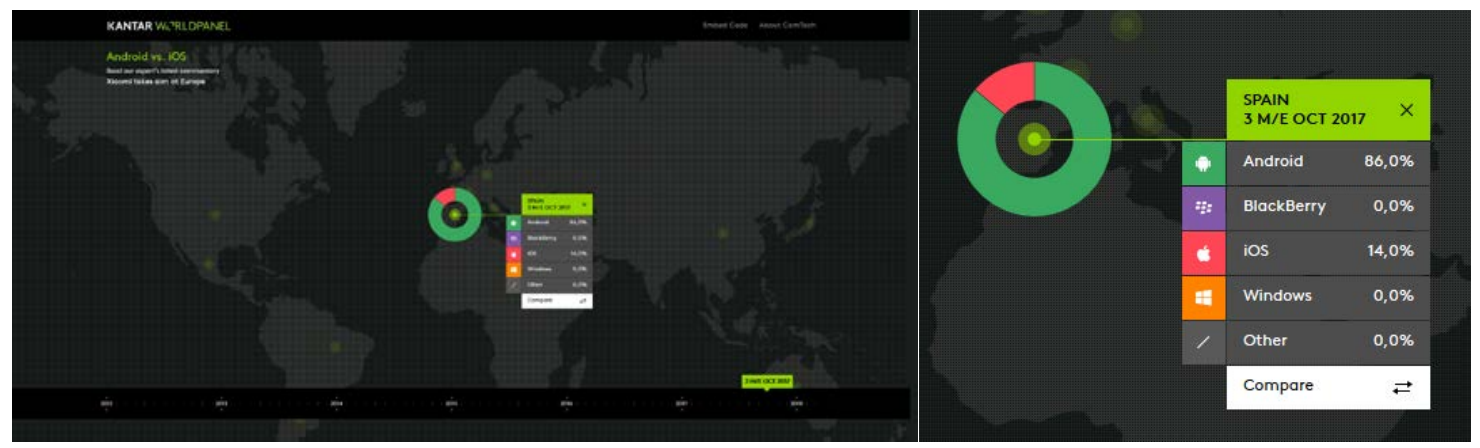

Figura 1. Sistemas operativos de smartphones en España (Octubre de 2017).

Fuente: https://www.kantarworldpanel.com/global/smartphone-os-market-share/

Un segundo parámetro a emplear para el proceso de selección ha sido la utilización en tiempo-real de la aplicación: esta condición permite una comunicación a tiempo real dentro del interfaz estudiante-profesor. De esta manera, las cuestiones planteadas en vivo por el profesor son contestadas individualmente por los alumnos de manera inmediata, dejando al alumno un tiempo prudencial para la respuesta.

El tercer parámetro en la selección ha sido el coste económico de la herramienta virtual. Se ha priorizado la elección de una herramienta o aplicación cuyo uso por parte del alumnado sea gratuito, de tal manera que el desarrollo de la actividad no conlleve ningún tipo de gasto para los estudiantes. 
El idioma en el que se desarrolla la aplicación no ha supuesto un factor determinante para su elección, dado que los alumnos a los que se destina esta herramienta se encuentran matriculados en los últimos cursos del Grado y disponen de una formación básica o avanzada en el lenguaje habitual de este tipo de aplicaciones (inglés).En caso de incorporar una tabla se hará del modo que se expresa a continuación.

\subsection{Selección de aplicaciones móviles}

Basándose en las premisas descritas, entre las herramientas disponibles en el mercado se han elegido las siguientes:

- Kahoot (https://kahoot.com/). Se trata de una plataforma de aprendizaje mixto basada en el juego, permitiendo a los docentes y estudiantes investigar, crear, colaborar y compartir conocimientos. Se puede acceder a la plataforma mediante ordenadores 0 a través de dispositivos móviles, de manera individual o en grupo. Puede emplearse con uso privado y/o cerrado para una clase o grupo de alumnos. El acceso puede hacerse a través de un navegador, o a través de la app "Kahoot!" para iOS y Android.

-iSpringSuite (https://www.ispringsolutions.com). Si se realiza una presentación previa en PowerPoint sobre la visita, este software permite convertirlo a html5, disponible en todos los navegadores. También permite la preparación de cuestionarios con gran variedad de tipos de cuestiones en html5. Los ficheros resultantes pueden verse en dispositivos móviles y descargarse través de la app gratuita "iSpringPlay", para iOS y Android.

\section{RESULTADOS}

\subsection{Implementación de la aplicación móvil "Kahoot"}

Respecto a la herramienta Kahoot, la aplicación permite crear cuestionarios a través de un proceso sencillo y guiado. En dos simples pasos consecutivos se inicia el cuestionario y se procede a generar las preguntas que desee formular el docente. El primer paso consiste en elegir el tipo herramienta a crear, de entre cuatro posibles opciones a elegir: cuestionario, puzle, debate y encuesta (Figura 2.a). En el presente proyecto se ha seleccionado la opción de cuestionario.
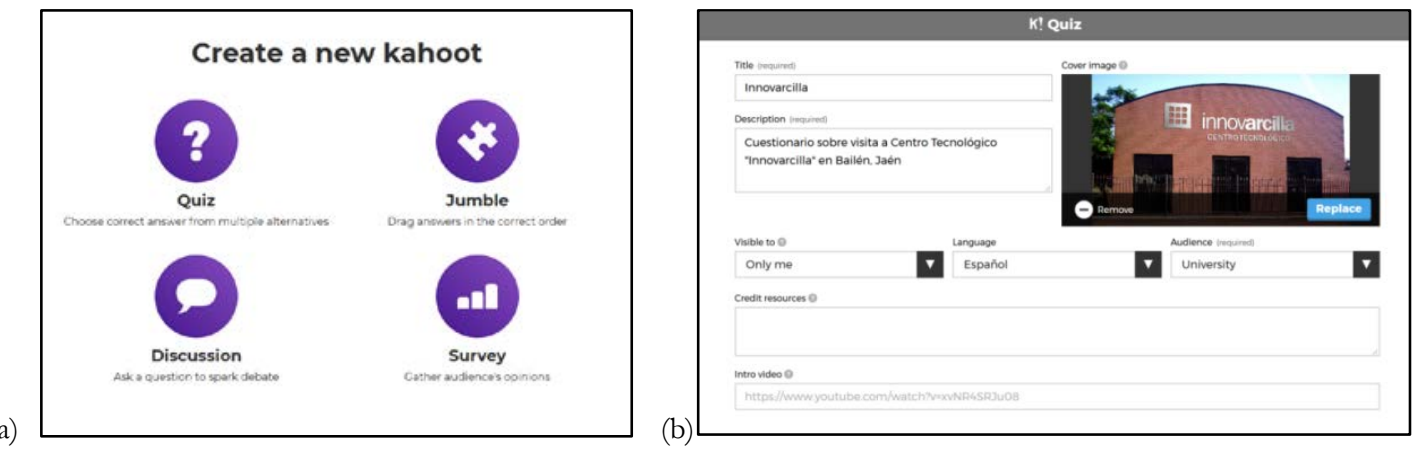

Figura 2. Paso iniciales de creación del cuestionario en aplicación Kahoot: (a) Elección del tipo de test y (b) Datos del test.

En un segundo paso consecutivo se detallan los datos del cuestionario creado, como el título, descripción, el lenguaje, así como aspectos visuales del mismo (Figura 2.b). En las siguientes etapas se procede a generar las preguntas del cuestionario. Para 
ello, desde la página general del cuestionario se pueden añadir cuántas cuestiones se estimen oportunas para completar el test (Figura 3.a).

(a)
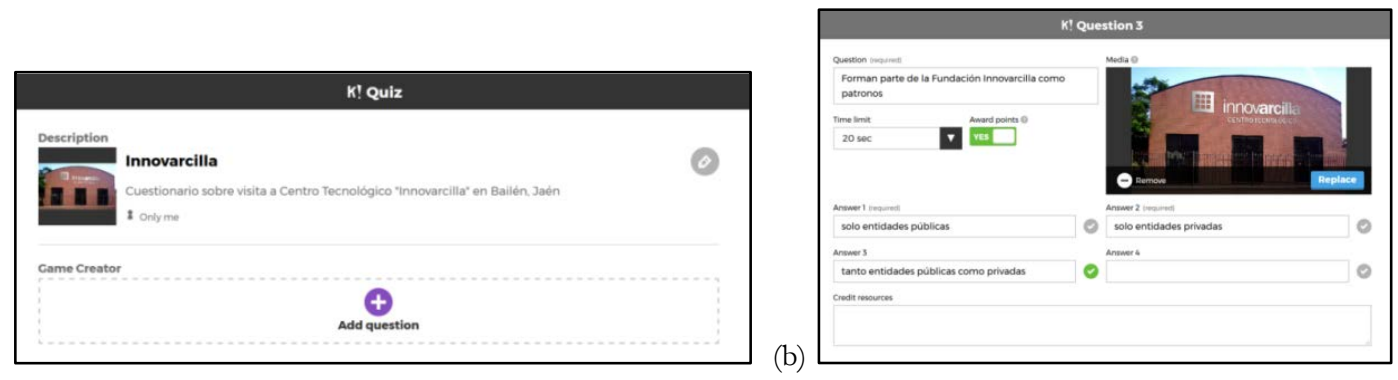

Figura 3. Generación de cuestiones en aplicación Kahoot: (a) Adición de la pregunta y (b)

Datos del test.

La creación de cada pregunta supone en una etapa adicional. Tal como se recoge en la Figura 3.b, algunos campos obligatorios así como opcionales pueden ser completados para generar cada nueva cuestión. La pregunta al alumno es el primer campo obligatorio, añadiendo el tiempo límite para la respuesta del alumno, así como un posible premio extra en la contabilización del cuestionario. Como mínimo dos posibles respuestas deben ser completadas por el docente para ofrecer al alumno, permitiendo la aplicación disponer de cuatro ítems al respecto. Una imagen específica puede ser añadida a la pregunta para ilustrar la cuestión o para la ayuda del alumno. Una vez creadas todas las preguntas que requiera el docente, el test presentará un formato de edición como el recogido en la Figura 4. En este punto pueden ser modificadas cada una de las preguntas o el orden de las mismas dentro del cuestionario general.

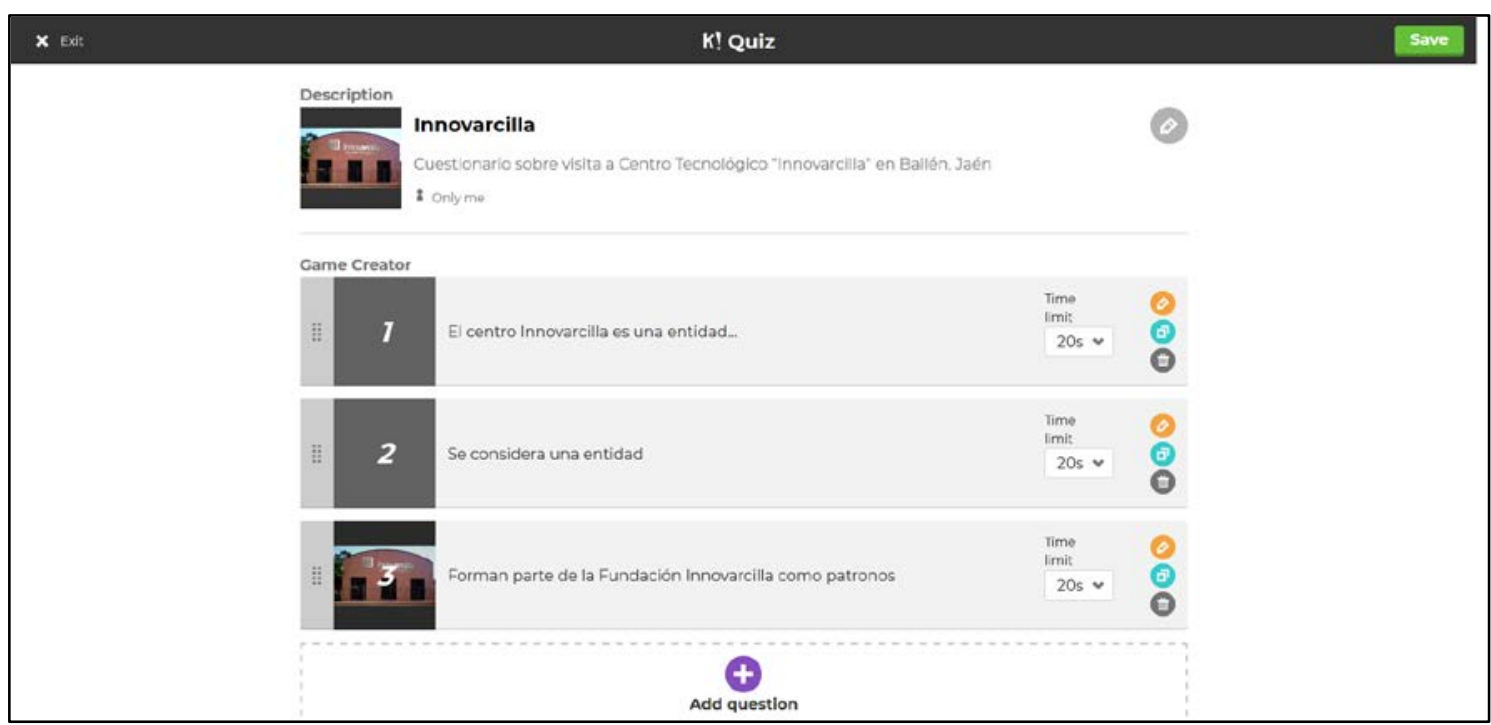

Figura 4. Formulario de edición del cuestionario.

Una vez creado todo el cuestionario y previo a la utilización de la aplicación como instrumento virtual de evaluación, se han llevado a cabo una serie de pruebas y test para comprobar la idoneidad de la misma de cara a cumplir los objetivos previstos. Para esto, solamente hay que pulsar el enlace a "Play" del test, generando un código 
numérico. Para ello el profesorado participante en el Proyecto ha simulado su actuación como alumnos en la actividad de evaluación en diferentes pruebas de ensayo. Los resultados obtenidos en estos ensayos han ayudado a depurar los posibles problemas surgidos en el proceso de evaluación con el uso de esta herramienta. Asimismo ha sido comprobada la facilidad de utilización por el docente de los datos recogidos por la aplicación.

Una vez testeada la aplicación en un entorno docente, se ha llevado a cabo la implementación como herramienta virtual de evaluación de los estudiantes. Para ello, antes de la realización de la actividad fuera del aula se indicó a los alumnos a través de Moodle las instrucciones para descargar la aplicación en función del sistema operativo que dispongan en su dispositivo móvil. Se procedió a realizar una prueba en aula para asegurarse que los estudiantes comprendían correctamente el sistema de funcionamiento de la herramienta en cuestión. Tanto en la sesión presencial como en el anuncio de convocatoria de la visita generado como novedad en Moodle, se les recordó la necesidad de llevar consigo dicho dispositivo (tablet o smartphone) el día de la actividad debidamente cargado.

La implementación de la aplicación en un entorno real se desarrolló durante una de las actividades fuera del aula programadas en la asignatura "Materiales Inorgánicos en la Industria Química", asignatura optativa del cuarto curso del Grado de Química. Se trataba de una visita al Centro de Innovación Tecnológica INNOVARCILLA, situado en la localidad de Bailén (Jaén). A la hora de programar la visita a empresa, el docente reservó el transporte apropiado en función del número de alumnos matriculados en la asignatura y asistentes a la actividad (20). Para la aplicación de este Proyecto, además del factor anterior, se tuvo en cuenta que el medio de transporte ofreciese conexión Wifi gratuita para los pasajeros. Este requisito se consideró imprescindible a la hora de contratar el medio de transporte ya que se aseguraría la completa conexión de todos los estudiantes presentes en la actividad, independientemente de que dispusieran o no de conexión de datos contratada con su operadora de telefonía.

De acuerdo con la planificación prevista, una vez finalizada la visita a la instalación industrial comenzó el proceso de evaluación con la herramienta virtual seleccionada previamente, Kahoot. Una vez que los alumnos se encontraron dispuestos en el vehículo para el trayecto de vuelta, el docente procedió con la aplicación y trasmitió a los alumnos el código numérico requerido (Figura 5) para acceder al cuestionario preguntas relativas a la visita que acaban de completar. Una vez que todos los alumnos (apareciendo como players en la aplicación) se incorporaron al test, el docente inició el cuestionario a través del enlace "Star". Desde este momento los alumnos disponían del tiempo fijado en cada pregunta para responder todo el test. 


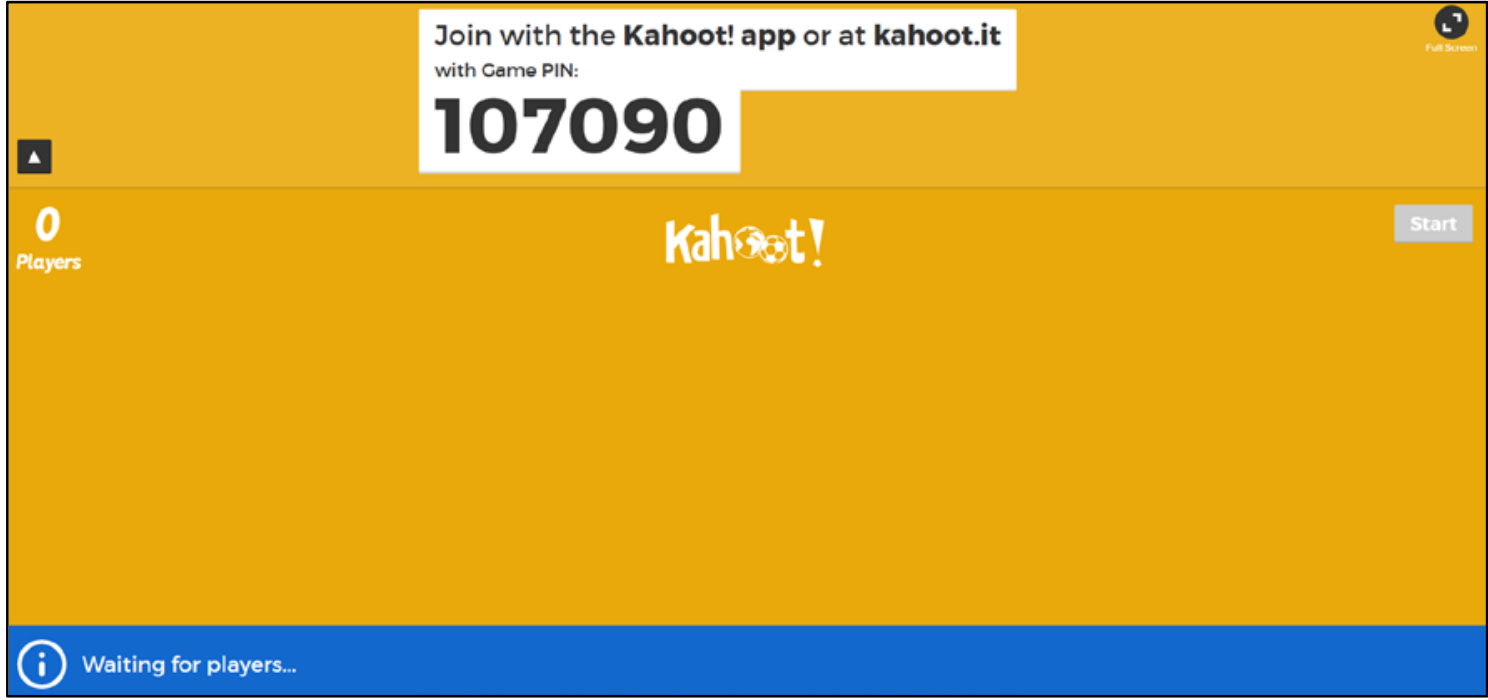

Figura 5. Página de inicio del cuestionario con el código numérico.

A modo de ejemplo, en la Figura 6 se presenta una de las preguntas del cuestionario durante un test de prueba para analizar la visualización correcta y la compabitibilidad con diferentes sistemas operativos (iOS y Android).

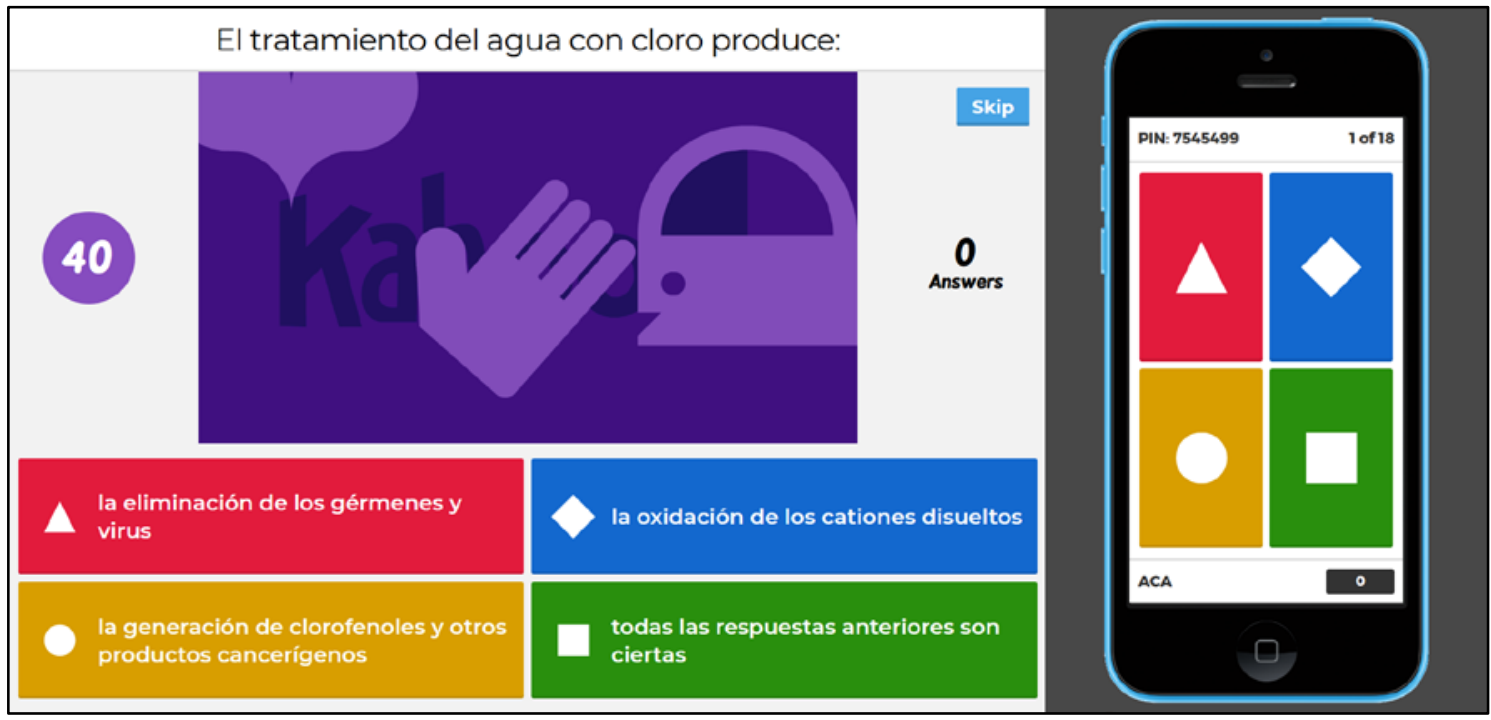

Figura 6. Ejemplo de pregunta en cuestionario durante el funcionamiento de la aplicación Kahoot.

Con las respuestas individuales de los alumnos, la aplicación genera inmediatamente una estadística colectiva en un fichero en formato de hoja de cálculo. En una primera aproximación a los resultados se presenta una vista general de los mismos, en un formato resumen el porcentaje total de respuestas acertadas y falladas por los alumnos (Figura 7). 


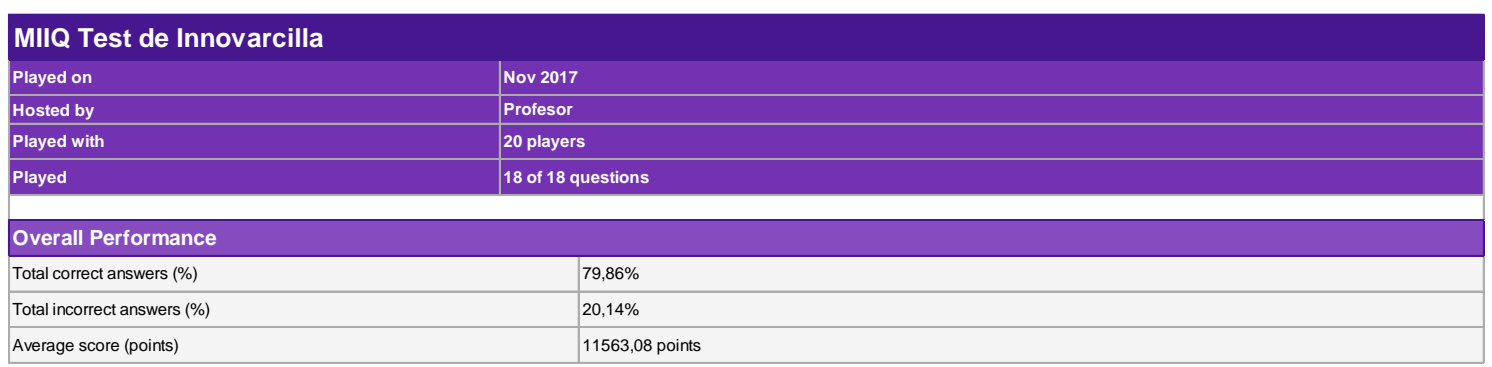

Figura 7. Resumen estadístico de los resultados del cuestionario con aplicación Kahoot.

En una segunda hoja de cálculo es generada una clasificación de los alumnos en función de la calificación obtenida en el cuestionario (Figura 8, donde se han eliminado los nombre de los alumnos por privacidad). Tres parámetros se tienen en cuenta a la hora de calcular los puntos asignados de cada alumno: respuestas acertadas, respuestas erróneas y tiempo de respuesta.

\begin{tabular}{|c|c|c|c|c|}
\hline \multicolumn{5}{|c|}{ MIIQ Test de prueba } \\
\hline \multicolumn{5}{|c|}{ Final Scores } \\
\hline Rank & Players & Total Score (points) & Correct Answers & Incorrect Answers \\
\hline 1 & & 24538 & 18 & 0 \\
\hline 2 & & 20770 & 15 & 0 \\
\hline 3 & & 20038 & 16 & 2 \\
\hline 4 & & 19484 & 16 & 2 \\
\hline 5 & & 16654 & 14 & 4 \\
\hline 6 & & 14700 & 13 & 5 \\
\hline 7 & & 7192 & 8 & 10 \\
\hline 8 & & 6937 & 6 & 0 \\
\hline 9 & & 3752 & 4 & 2 \\
\hline 10 & & 1914 & 2 & 1 \\
\hline 11 & & 1805 & 2 & 0 \\
\hline 12 & & 973 & 1 & 3 \\
\hline
\end{tabular}

Figura 8. Tabla de calificaciones del cuestionario con aplicación Kahoot.

En una tercera hoja de cálculo se pueden identificar las respuestas de cada alumno para todas las preguntas (Figura 9). Este mismo documento también contiene la puntuación recibida por pregunta para el alumno. 


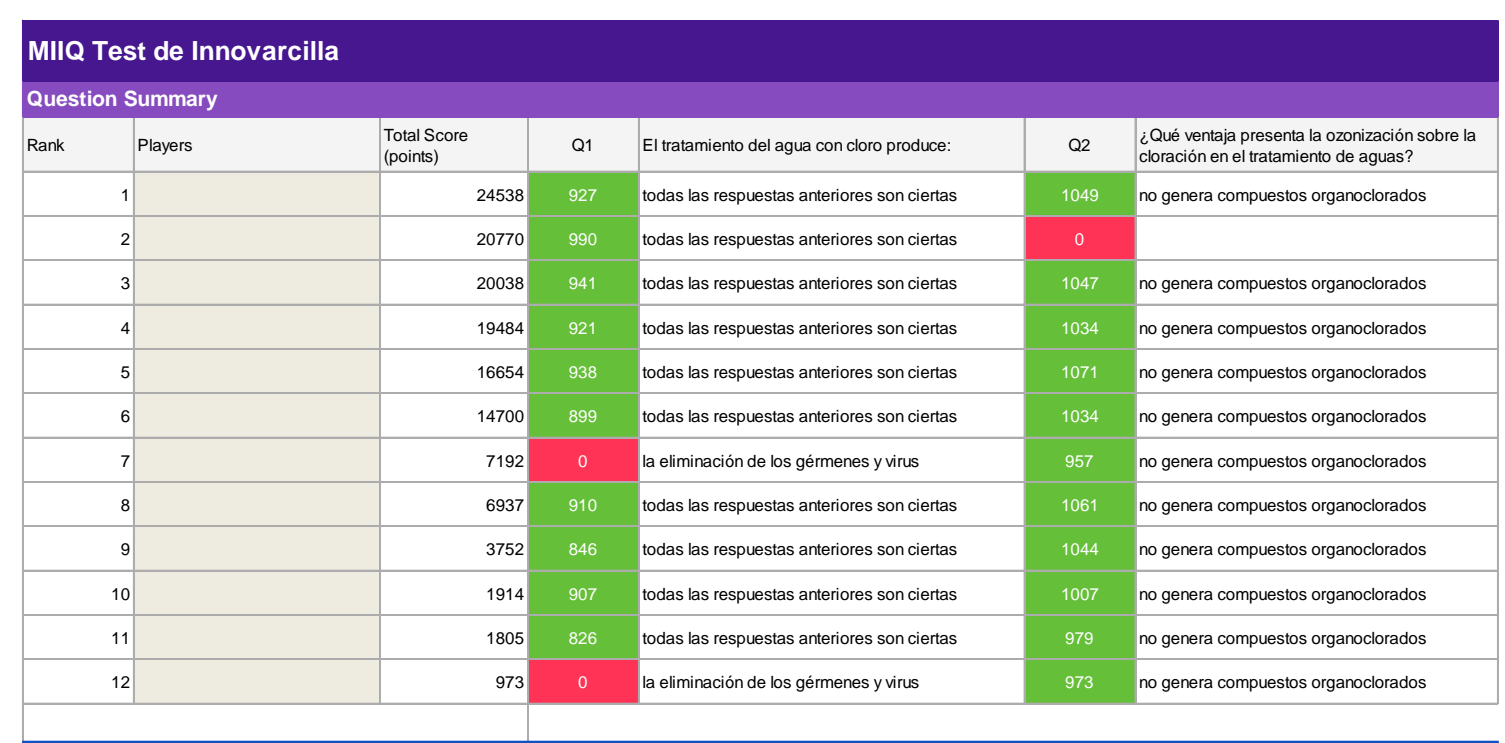

Figura 9. Tabla de resumen de preguntas por alumno del cuestionario con aplicación Kahoot.

Toda la información estadística generada en este cuestionario permite identificar los puntos flacos de comprensión por parte del alumnado, de manera que el profesor, a tiempo real, puede incidir en dichos aspectos. De la acumulación de las respuestas acertadas por cada alumno y de la puntuación global se extrae el porcentaje de éxito, empleándose este dato como calificación de la actividad realizada en la ponderación estimada dentro de la evaluación de la asignatura.

La implementación del proyecto ha generado un significativo impacto en el proceso de aprendizaje del alumnado y en la satisfacción del mismo. En la Figura 10 se recogen los principales parámetros que se han visto afectados por el uso de la herramiento virtual. A través de las encuestas realizadas al alumnado sobre la evaluación de la actividad docente del profesorado se puede observar el incremento de dos parámetros claves: "Adecuación de los sistemas de evaluación" y "Utilización de recursos didácticos que facilitan el aprendizaje". Teniendo en cuenta que estos parámetros se puntuan en escala de $0-5$, en ambos casos, se logra incrementar un 10 $\%$ el grado de satisfacción del alumno. Igualmente, la implementación de esta plataforma innovativa como herramienta de evaluación de las actividades fuera del aula ha supuesto una mejora en las calificaciones obtenidas por el alumnado en este apartado. Este proceso de virtualización ha permitido a los alumnos elevar la calificación media en la evaluación final de la asignatura. 


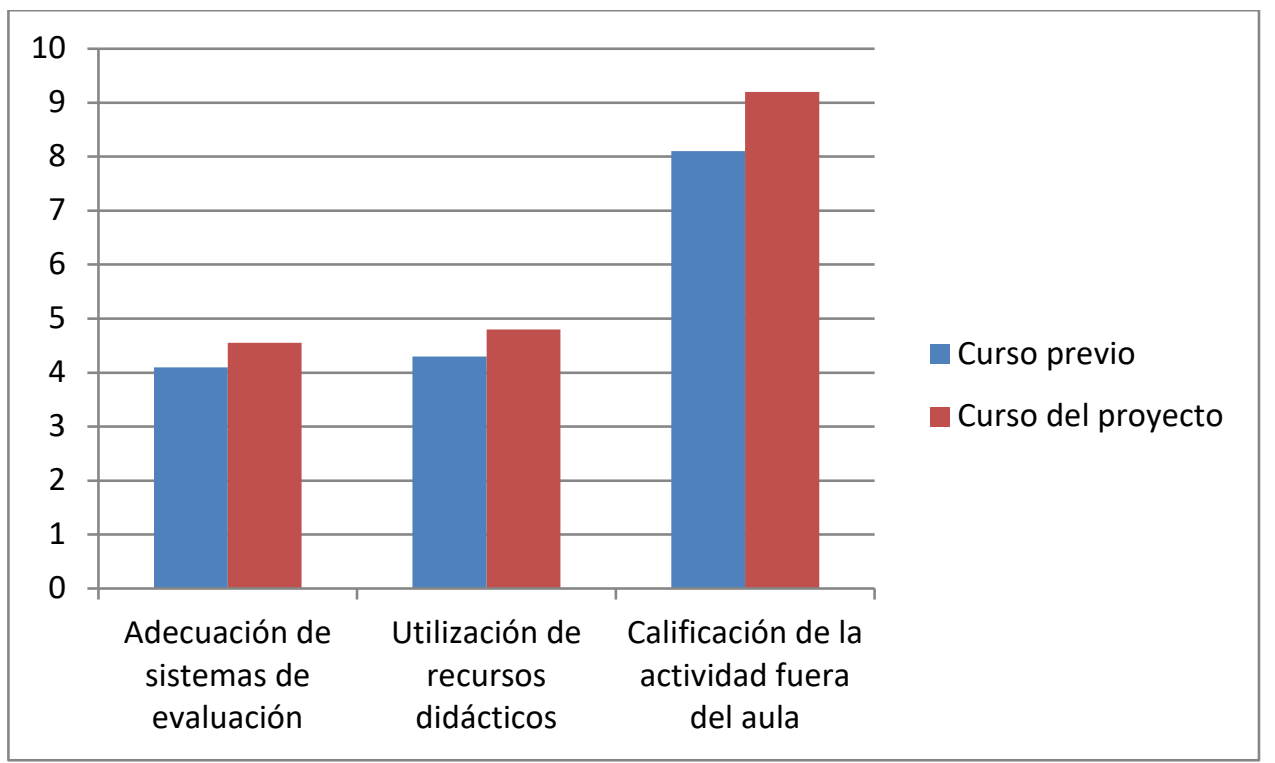

Figura 10. Resultados de implementación del proyecto de innovación sobre el aprendizaje y satisfaccion del alumnado.

Los resultados logrados con la implementación de esta plataforma virtual demuestran la mejora significativa en la transferencia del conocimiento dentro del proceso de enseñanza-aprendizaje. El uso de una herramienta como Kahoot permite potenciar la consolidación de los conocimientos adquiridos durante la actividad desarrollada fuera del aula, así como actuar de instrumento evaluador facilitando al profesorado la actividad docente.

\subsection{Implementación de la plataforma "iSpringSuite"}

La plataforma iSpringSuite ha sido empleada para la creación de cuestionarios en un sentido similar al descrito en el apartado anterior. Se han desarrollado cuestionarios de seguimiento en la asignatura "Gestión Energética y Ecoeficiencia", correspondiente al tercer cursos del grado de Ciencias Ambientales, y que se imparte en el segundo cuatrimestre. Estos cuestionarios se han utilizado en cuatro casos:

- Visita a la cementera Cosmos, en Córdoba.

- Visita a la planta Gemasolar, en Fuentes de Andalucía (Sevilla).

- Visita a la Central Térmica de Viesgo, en Puente Nuevo (Córdoba).

- Curso de Gestión de Residuos Radioactivos, organizados por "Jóvenes Nucleares", que este curso remplaza a la visita al Almacén de Residuos Radioactivos del Cabril.

En la Figura 11 se recogen las presentaciones iniciales de cada uno de los cuestionarios creados en la iSpringSuite, implementados en las cuatro visitas citadas. 


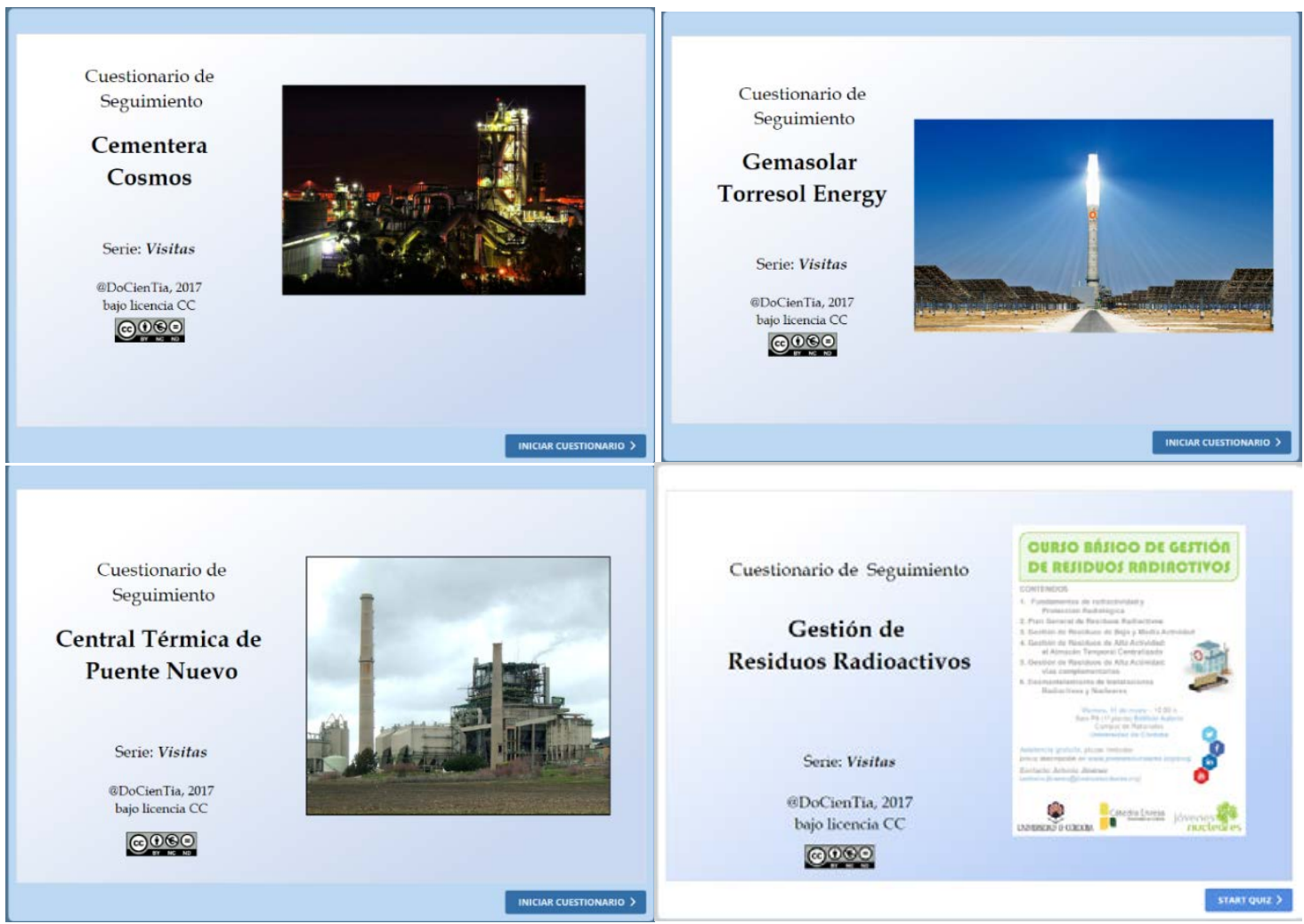

Figura 11. Pantalla inicial de los cuestionarios con plataforma iSpringSuite.

Actualmente se está también trabajando en la elaboración de cuestionarios para las visitas que se realizan en la asignatura "Química, Historia y Sociedad", que se imparte en el cuarto curso del grado de Química, como por ejemplo las visitas al yacimiento arqueológico de "Medina Azahara" y el "Museo Arqueológico" de Córdoba.

\section{CONCLUSIONES}

Gracias al proyecto desarrollado y la implementación de las herramientas virtuales se ha logrado facilitar a docentes y estudiantes el proceso de evaluación de las actividades fuera del aula, especialmente, de las visitas a empresas. Adicionalmente, se maximiza el aprovechamiento del periodo de tiempo dedicado al desplazamiento desde el centro de origen hasta la empresa objetivo de la visita y el retorno una vez finalizada la actividad en la industria. Gracias a la virtualización, se hace más atractivo al alumnado la adquisición de competencias asociadas a las actividades fuera del aula, dado el empleo de herramientas visualmente más favorables. En este sentido se ha logrado potenciar la virtualización de los procesos de evaluación de los estudiantes, especialmente, los dedicados a la sustitución de herramientas tradicionales tales como la elaboración de memorias escritas.

De acuerdo con los resultados y el éxito logrado en la implementación de las herramientas virtuales de evaluación, estas aplicaciones serán empleadas en los próximos cursos académicos en las asignaturas donde se ha desarrollado este proyecto ("Materiales Inorgánicos en la Industria Química" y "Gestión Energética y Ecoeficiencia"). Asimismo se prevé su implementación en otras asignaturas donde 
participan los docentes implicados en este proyecto, tales como "Química, Historia y Sociedad" y "Contaminación por metales pesados".

\section{REFERENCIAS}

Canós, L., \& Mauri, J. (2005). Metodologías activas para la docencia y aplicación de las nuevas tecnologías: una experiencia. XX Simposium Nacional de la URSI, Gandía (Valencia).

Canós, L., Canós, M.J., \& Liern, V. (2008). El uso de las nuevas tecnologías aplicadas a la educación superior. XVII Jornadas ASEPUMA-V Encuentro Internacional Rect@, 17(1), 612.

Guenaga, G., \& Hernando, G. (2012). Visita a empresa: una herramienta educativa para la universidad y una herramienta de marketing para la empresa. Revista Turismo \& Desenvolvimento, 1, 77-92.

Ghemawat, P. (2017). Strategies for higher education in the digital age. California Management Review, 59(4), 56-78.

Guardia, J., Del Olmo, J., Roa, I., \& Berlanga, V. (2019). Innovation in the teachinglearning process: the case of Kahoot!. On the Horizon, 27 (1), 35-45.

Gündüz, A.Y., \& Akkoyunlu, B. (2019). The Gamification Tool For The Classroom Response Systems: Kahoot. Hacettepe University Journal of Education. 1-10.

Martins E.R., Geraldes W.B., Afonseca U.R., \& Gouveia L.M.B. (2019). Using Kahoot as a Learning Tool. En I. Ramos, R. Quaresma, P., Silva \& T. Oliveira. (eds) Information Systems for Industry 4.0. Lecture Notes in Information Systems and Organisation, 31. Springer.

Mohamed, M., Perez, M.A., \& Montero, M.A. (2017). Salidas pedagógicas como metodología de refuerzo en la Enseñanza Secundaria. ReiDoCrea, 6, 194-210.

Pons, P., Bravo, C., \& Ramírez, G. (2016). La enseñanza universitaria apoyada en plataformas virtuales. Cambios en las prácticas docentes: el caso de la Universidad de Sevilla. Estudios sobre Educación, 20, 23-48. 